\title{
Probe of the Randall-Sundrum-like model with the small curvature via light-by-light scattering at the LHC
}

\author{
S. C. İnan ${ }^{*}$ \\ Department of Physics, Sivas Cumhuriyet University, 58140, Sivas, Turkey \\ A. V. Kisselev (1) \\ A.A. Logunov Institute for High Energy Physics, NRC “Kurchatov Institute”, \\ 142281, Protvino, Russian Federation
}

(Received 7 June 2019; published 5 November 2019)

\begin{abstract}
The LHC possibilities to constrain the parameters of the Randall-Sundrum-like model with one warped extra dimension and small curvature through the diphoton production in the photon-induced process $p p \rightarrow p \gamma \gamma p \rightarrow p^{\prime} \gamma \gamma p^{\prime}$ are investigated. The acceptance of the forward detectors $0.015<\xi<0.15$, where $\xi$ is the fractional proton momentum loss of the incident protons, is considered. The sensitivity bounds on the five-dimensional gravity scale are obtained as a function of the LHC integrated luminosity.
\end{abstract}

DOI: 10.1103/PhysRevD.100.095004

\section{INTRODUCTION}

The Standard Model (SM), which defines the fundamental particles and their interactions at the electroweak energy scale, has been proven in all experiments, including the LHC. Nevertheless, scientists are still searching for solutions for many problems for which the SM cannot give a satisfactory solution. The hierarchy problem, which involves the large energy gap between the electroweak scale and the gravity scale, is one of these problems. The most important answers to this unexplained phenomenon can be given by beyond the SM theories, which include additional dimensions. Therefore, such models have attracted much attention in recent years, and many articles have been published in the literature.

At hadron colliders, inelastic collisions are generally performed, and their results are examined. However, the hadron colliders can also be used as photon-photon, photon-proton colliders as applied in the Tevatron $[1,2]$ and LHC [3-8]. The current results that are found in these experiments are in agreement with theoretical expectations. Specifically, the LHC experiments have shown that such photon-induced processes are an important search for new physics. The most important advantage of the photoninduced process is that it has a clean background. It is

\footnotetext{
sceminan@cumhuriyet.edu.tr

† alexandre.kisselev@ihep.ru
}

Published by the American Physical Society under the terms of the Creative Commons Attribution 4.0 International license. Further distribution of this work must maintain attribution to the author(s) and the published article's title, journal citation, and DOI. Funded by SCOAP. because this process does not include a lot of QCD originating backgrounds and uncertainties resulting from proton dissociation into jets. All these backgrounds make it difficult to identify the new physics signal beyond the SM (BSM). The photon-photon collisions through the process $p p \rightarrow p \gamma \gamma p \rightarrow p^{\prime} X p^{\prime}$ have very little background. The schematic diagram for this collision is shown in Fig. 1. As one can see, both protons remain intact in this exclusive process.

Examining photon-photon interactions at the LHC is possible thanks to the plan prepared by the ATLAS Forward Physics (AFP) Collaboration and joint CMSTOTEM Precision Proton Spectrometer (CT-PPS) [9-12]. These plans include forward detectors that are placed symmetrically at a distance from the main detectors. The forward detectors have charged particle trackers. They can catch the intact protons after elastic photon emission in the interval $\xi_{\min }<\xi<\xi_{\max }$, where $\xi$ is the fractional proton momentum loss of the protons, $\xi=\left(|\vec{p}|-\left|\vec{p}^{\prime}\right|\right) /|\vec{p}|$. Here, $\vec{p}$ is the incoming proton momentum, and $\vec{p}^{\prime}$ is

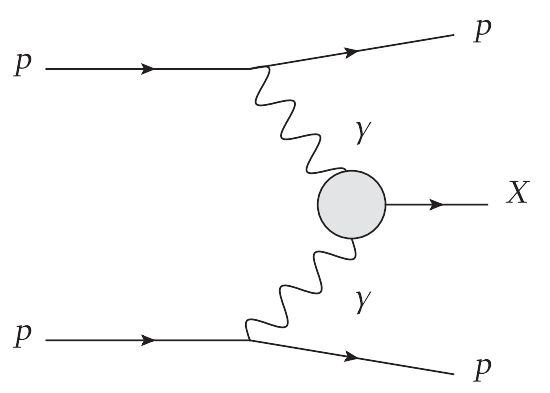

FIG. 1. Schematic diagram for the reaction $p p \rightarrow p \gamma \gamma p \rightarrow$ $p^{\prime} X p^{\prime}$. In our case, $X=\gamma \gamma$. 
the momentum of the intact scattered proton. The application of forward detectors to detect the scattered protons is used to identify the collision kinematics, and consequently, photon-induced processes can be studied at the LHC. Forward detectors should be installed closer to the main detectors to achieve greater values of $\xi$.

AFP has the $0.015<\xi<0.15$ detector acceptance range [11]. The detector acceptance range of the CT-PPS is similar [12]. AFP includes two types of studies. The first one is exploratory physics (anomalous couplings between $\gamma$ and $Z$ or $W$ bosons, exclusive production, etc.). The second one is the SM physics (double Pomeron exchange, exclusive production in the jet channel, single diffraction, $\gamma \gamma$ physics, etc.). The main goal of the CT-PPS experiment is to perform measurements at high luminosity, such as gamma-gamma scattering. These charged particle detectors enable us to determine almost all inelastic interactions in the forward area. In this way, a very wide solid angle can be examined with the support of the CMS detector. Also, the forward detectors can be applied for precise studies [13-15]. Pile-up events can occur as a result of such high-luminosity and high-energy interactions. However, by using kinematics, timing constraints, and exclusivity conditions, these backgrounds can be extremely restricted $[16,17]$. There are many phenomenological papers in the literature that are based on the photon-induced reactions at the LHC aimed at searching for the BSM physics [18-41].

In the present paper, we investigate the RandallSundrum-like model scenario with the small curvature (the details are given in Sec. III) through the main process $p p \rightarrow p \gamma \gamma p \rightarrow p^{\prime} \gamma \gamma p^{\prime}$ with the subprocess $\gamma \gamma \rightarrow \gamma \gamma$ in this study for the acceptance $0.015<\xi<0.15$. The first evidence for $\gamma \gamma \rightarrow \gamma \gamma$ scattering was observed by the ATLAS Collaboration in high-energy ultraperipheral heavy ions collisions [42]. After that, the CMS Collaboration reported the same process [43]. Therefore, studies on this process have gained more importance in recent times. Recently, we have studied the photon-induced dimuon production at the LHC [44]. It is clear that any BSM scenario must be checked in a variety of processes in order to find the most appropriate one. As we will see below, the bounds on the main parameters of the model for the diphoton production are better than the bounds obtained in Ref. [44]. Note that the process going through the subprocess $\gamma \gamma \rightarrow \gamma \gamma$ is known to be one of the most clean channels.

The processes contributing to the SM exclusive photonphoton production consist of diagrams with charged fermions (leptons, quarks), $W$ boson loop contributions, and gluon loop diagrams. Also, the interference terms of these processes should be taken into account in order to obtain the whole SM cross section.

These processes have been examined in Refs. [45-49]. QCD gluon loop contributions are dominant at low energies, whereas at high energies, $W$ loop contributions dominate. As shown in Ref. [50], the QCD loop contribution can be neglected for the diphoton mass larger than $200 \mathrm{GeV}$. In our study, we have implemented the cut on the diphoton mass of $200 \mathrm{GeV}$, and therefore we have omitted the QCD loop contributions.

There are 16 helicity amplitudes of the subprocess $\gamma \gamma \rightarrow \gamma \gamma$. However, if $\mathrm{T}$ invariance, $\mathrm{P}$ invariance, and Bose statistics are taken into consideration, the following relations are obtained:

$$
\begin{aligned}
M_{++++} & =M_{----}, \quad M_{++--}=M_{--++}, \\
M_{+-+-} & =M_{-+-+}, \quad M_{+--+}=M_{-++-}, \\
M_{+++-} & =M_{++-+}=M_{+-++}=M_{-+++} \\
& =M_{---+}=M_{--+-}=M_{-+--}=M_{+---} .
\end{aligned}
$$

With using there relations, the total matrix element takes the form

$$
\begin{aligned}
|M|^{2}= & 2\left|M_{++++}\right|^{2}+2\left|M_{++--}\right|^{2}+2\left|M_{+-+-}\right|^{2} \\
& +2\left|M_{+--+}\right|^{2}+8\left|M_{+++-}\right|^{2} .
\end{aligned}
$$

Taking into account the crossing symmetry, we find relations between amplitudes,

$$
\begin{aligned}
& M_{+-+-}(\hat{s}, \hat{t}, \hat{u})=M_{++++}(\hat{u}, \hat{t}, \hat{s}), \\
& M_{+--+}(\hat{s}, \hat{t}, \hat{u})=M_{++++}(\hat{t}, \hat{s}, \hat{u})=M_{++++}(\hat{t}, \hat{u}, \hat{s}), \\
& M_{+--+}(\hat{s}, \hat{t}, \hat{u})=M_{+-+-}(\hat{s}, \hat{u}, \hat{t}) .
\end{aligned}
$$

All of the SM helicity amplitudes can be found in Refs. [47,48]. Using relations $\ln (\hat{u})=\ln (-\hat{u})+i \pi$, $\ln (\hat{t})=\ln (-\hat{t})+i \pi, \quad \ln (-\hat{s})=\ln (\hat{s})+i \pi, \quad$ the helicity amplitudes corresponding to the fermion loops can be obtained by neglecting the terms like $m_{f}^{2} / \hat{s}, m_{f}^{2} / \hat{t}$ and $m_{f}^{2} / \hat{u}$

$$
\begin{aligned}
& \frac{1}{\alpha^{2} Q_{f}^{4}} M_{++++}^{f}(\hat{s}, \hat{t}, \hat{u}) \\
& =-8-8\left(\frac{\hat{u}-\hat{t}}{\hat{s}}\right) \operatorname{Ln}\left(\frac{\hat{u}}{\hat{t}}\right) \\
& \quad-4\left(\frac{\hat{t}^{2}+\hat{u}^{2}}{\hat{s}^{2}}\right)\left[\operatorname{Ln}\left(\frac{\hat{u}}{\hat{t}}\right) \operatorname{Ln}\left(\frac{\hat{u}}{\hat{t}}\right)+\pi^{2}\right], \\
& M_{+++-}^{f}(\hat{s}, \hat{t}, \hat{u}) \simeq M_{++--}^{f}(\hat{s}, \hat{t}, \hat{u}) \simeq 8 \alpha^{2} Q_{f}^{4},
\end{aligned}
$$

where invariant Mandelstam variables are defined as $\hat{s}=\left(p_{1}+p_{2}\right)^{2}, \hat{t}=\left(p_{1}-p_{3}\right)^{2}$, and $\hat{u}=\left(p_{2}-p_{3}\right)^{2}$ and $m_{f}$ and $Q_{f}$ are the mass of the fermion $f$ and its charge, respectively. The other helicity amplitudes can be obtained by using relations in Eq. (3).

Using a similar approximation and neglecting the terms $m_{W}^{2} / \hat{s}, m_{W}^{2} / \hat{t}$ and $m_{W}^{2} / \hat{u}$, the $W$ loop contribution can be found to be 


$$
\begin{aligned}
& \frac{1}{\alpha^{2}} M_{++++}^{W}(\hat{s}, \hat{t}, \hat{u})=-16 i {\left[\frac{\hat{s}}{\hat{t}} \operatorname{Ln}\left(\frac{-\hat{t}}{m_{W}^{2}}\right)+\frac{\hat{s}}{\hat{u}} \operatorname{Ln}\left(\frac{-\hat{u}}{m_{W}^{2}}\right)\right]+12+12\left(\frac{\hat{u}-\hat{t}}{\hat{s}}\right) \operatorname{Ln}\left(\frac{\hat{u}}{\hat{t}}\right)+16\left(1-\frac{3 \hat{t} \hat{u}}{4 \hat{s}^{2}}\right)\left[\operatorname{Ln}\left(\frac{\hat{u}}{\hat{t}}\right) \operatorname{Ln}\left(\frac{\hat{u}}{\hat{t}}\right)+\pi^{2}\right] } \\
&+ 16\left[\frac{\hat{s}}{\hat{t}} \operatorname{Ln}\left(\frac{\hat{s}}{m_{W}^{2}}\right) \operatorname{Ln}\left(\frac{-\hat{t}}{m_{W}^{2}}\right)+\frac{\hat{s}}{\hat{u}} \operatorname{Ln}\left(\frac{\hat{s}}{m_{W}^{2}}\right) \operatorname{Ln}\left(\frac{-\hat{u}}{m_{W}^{2}}\right)+\frac{\hat{s}^{2}}{\hat{t} \hat{u}} \operatorname{Ln}\left(\frac{-\hat{t}}{m_{W}^{2}}\right) \operatorname{Ln}\left(\frac{-\hat{u}}{m_{W}^{2}}\right)\right], \\
& \frac{1}{\alpha^{2}} M_{+-+-}^{W}(\hat{s}, \hat{t}, \hat{u})=-i \pi\left[12\left(\frac{\hat{s}-\hat{t}}{\hat{u}}\right)+32\left(1-\frac{3 \hat{t} \hat{s}}{4 \hat{u}^{2}}\right) \operatorname{Ln}\left(\frac{\hat{s}}{-\hat{t}}\right)+16 \frac{\hat{u}}{\hat{s}} \operatorname{Ln}\left(\frac{-\hat{u}}{m_{W}^{2}}\right)+16 \frac{\hat{u}^{2}}{\hat{t} \hat{s}} \operatorname{Ln}\left(\frac{-\hat{t}}{m_{W}^{2}}\right)\right]+12 \\
&+12\left(\frac{\hat{s}-\hat{t}}{\hat{u}}\right) \operatorname{Ln}\left(\frac{\hat{s}}{-\hat{t}}\right)+16\left(1-\frac{3 \hat{t} \hat{s}}{4 \hat{u}^{2}}\right) \operatorname{Ln}\left(\frac{\hat{s}}{-\hat{t}}\right) \operatorname{Ln}\left(\frac{\hat{s}}{-\hat{t}}\right)+16\left[\frac{\hat{u}}{\hat{t}} \operatorname{Ln}\left(\frac{-\hat{u}}{m_{W}^{2}}\right) \operatorname{Ln}\left(\frac{-\hat{t}}{m_{W}^{2}}\right)\right. \\
&\left.+\frac{\hat{u}}{\hat{s}} \operatorname{Ln}\left(\frac{-\hat{u}}{m_{W}^{2}}\right) \operatorname{Ln}\left(\frac{\hat{s}}{m_{W}^{2}}\right)+\frac{\hat{u}^{2}}{\hat{t} \hat{s}} \operatorname{Ln}\left(\frac{-\hat{t}}{m_{W}^{2}}\right) \operatorname{Ln}\left(\frac{\hat{s}}{m_{W}^{2}}\right)\right], \\
& M_{+-+-}^{W}(\hat{s}, \hat{t}, \hat{u})= M_{-+-+}^{W}(\hat{s}, \hat{t}, \hat{u}), \\
& M_{+++-}^{W}(\hat{s}, \hat{t}, \hat{u}) \simeq M_{++--}^{W}(\hat{s}, \hat{t}, \hat{u}) \simeq-12 \alpha^{2} . \quad(8) \quad \frac{d N_{\gamma}}{d E_{\gamma} d Q^{2}}=\frac{\alpha}{\pi} \frac{1}{E_{\gamma} Q^{2}}\left[\left(1-\frac{E_{\gamma}}{E}\right)\left(1-\frac{Q_{\min }^{2}}{Q^{2}}\right) F_{E}\left(Q^{2}\right)\right. \\
&\left.+\frac{E_{\gamma}^{2}}{2 E^{2}} F_{M}\left(Q^{2}\right)\right] .
\end{aligned}
$$

In case of $m_{W}^{2} \ll \hat{s}, W$ loop helicity amplitudes (especially, their imaginary parts) become dominant. In $m_{W}^{2} \gg \hat{s}$ energy region, fermion loop contributions are much bigger than the $W$ loop contributions. The contribution of the top quark in all energy regions is not taken into account since it is very small compared to other fermions and $W$ loop contributions [51].

\section{PHOTON-PHOTON INTERACTIONS AT THE LHC}

As was mentioned above, it is possible to examine the photon-photon interaction with using forward detectors at the LHC. After elastic photon emission with small angles and low transverse momentum, the protons deviate slightly from their paths along the beam pipe and are probed in the forward detectors without being detected by the main detectors. This deviation is related to $\xi$. Emitted photons, which are called almost-real photons, have very low virtualities. Therefore, these photons can be considered as on mass-shell photons. In this case, the process $p p \rightarrow$ $p \gamma \gamma p \rightarrow p^{\prime} X p^{\prime}$ occurs, and the final state $X=\gamma \gamma$ is measured by the central detector. The value of $\xi$ can be determined by using forward detectors. Hence, the centerof-mass energy of the $\gamma \gamma$ collision can be known. It is given as $W=2 E \sqrt{\xi_{1} \xi_{2}}$, where $E$ is the energy of the incoming protons with the mass $m_{p}$. The photon-photon interaction in the hadron collision can be studied with the equivalent photon approximation (EPA) $[52,53]$. The EPA includes a spectrum that depends on the photon energy $\left(E_{\gamma}=\xi E\right)$ and photon virtuality $\left(Q^{2}=-q^{2}\right)$ :
The minimal photon virtuality $Q_{\min }^{2}$ as well as electric $\left(F_{E}\right)$ and magnetic $\left(F_{M}\right)$ form factors of the proton in the above equation are defined in Ref. [51]. From this perspective, the resulting luminosity spectrum $d L^{\gamma \gamma} / d W$ is obtained as

$$
\begin{aligned}
\frac{d L^{\gamma \gamma}}{d W}= & \int_{Q_{1, \min }^{2}}^{Q_{\max }^{2}} d Q_{1}^{2} \int_{Q_{2, \min }^{2}}^{Q_{\max }^{2}} d Q_{2}^{2} \int_{y_{\min }}^{y_{\max }} d y \frac{W}{2 y} f_{1} \\
& \times\left(\frac{W^{2}}{4 y}, Q_{1}^{2}\right) f_{2}\left(y, Q_{2}^{2}\right),
\end{aligned}
$$

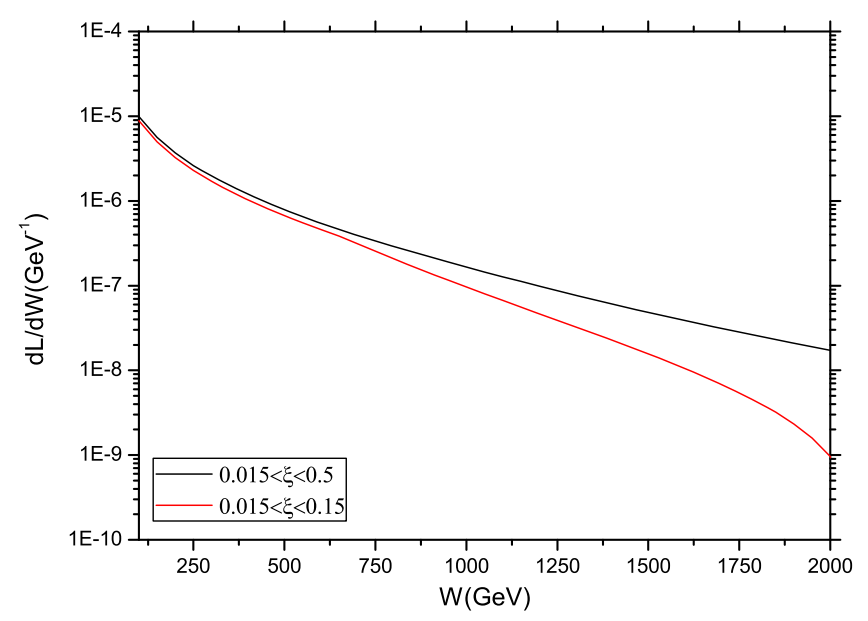

FIG. 2. Effective $\gamma \gamma$ luminosity as a function of the invariant mass of the two-photon system. The figure shows the effective luminosity for two forward detector acceptances, $0.015<\xi<$ 0.5 and $0.015<\xi<0.15$. 
with $\quad y_{\min }=\max \left(W^{2} /\left(4 \xi_{\max } E\right), \xi_{\min } E\right), \quad y_{\max }=\xi_{\max } E$, $f=d N /\left(d E_{\gamma} d Q^{2}\right), Q_{\max }^{2}=2 \mathrm{GeV}$. The contribution of more than this $Q_{\max }^{2}$ value to the integral (10) is negligible. In Fig. 2, we show the effective $\gamma \gamma$ luminosity as a function of $W$ for the detector acceptances $0.015<\xi<0.5$ and $0.015<\xi<0.15$. Using Eq. (10), the total cross section for the $p p \rightarrow p \gamma \gamma p \rightarrow p^{\prime} \gamma \gamma p^{\prime}$ can be given as

$$
d \sigma=\int \frac{d L^{\gamma \gamma}}{d W} d \hat{\sigma}_{\gamma \gamma \rightarrow \gamma \gamma}(W) d W
$$

where $d \hat{\sigma}_{\gamma \gamma \rightarrow \gamma \gamma}(W)$ is the cross section of the subprocess $\gamma \gamma \rightarrow \gamma \gamma$.

\section{RANDALL-SUNDRUM-LIKE MODEL WITH A SMALL CURVATURE}

One of the promising possibilities to go beyond the SM is to consider a scenario with extra spatial dimensions (EDs). A framework with EDs is motivated by (super)string theory [54]. One of the main goals of such theories is to explain the hierarchy relation between electromagnetic and Planck scales. In the model proposed by Arkani-Hamed et al. and Antoniadis et al. [55-57] (ADD), the hierarchy relation looks like

$$
\bar{M}_{\mathrm{Pl}}^{2}=V_{d} M_{D}^{d+2}
$$

where $V_{d}=\left(2 \pi R_{c}\right)^{d}$ is the volume of the compactified EDs with the size $R_{c}, \bar{M}_{\mathrm{Pl}}=M_{\mathrm{Pl}} / \sqrt{8 \pi}$ is the reduced Planck mass, and $M_{D}$ is the fundamental gravity scale in $D=4+d$ dimensions. The masses of the Kaluza-KLein (KK) gravitons in the ADD model are

$$
m_{n}=\frac{n}{R_{c}}, \quad n=\sqrt{n_{1}^{2}+n_{2}^{2}+\cdots n_{d}^{2}}
$$

where $n_{i}=0,1, \ldots(i=1,2, \ldots d)$. Thus, in the scenario with large EDs, the mass splitting $\Delta m_{K K}=1 / R_{c}$ is very small.

However, this solution of the hierarchy problems in the ADD model cannot be considered satisfactory, since formula (12) explains a large value of the Planck mass by introducing a new large scale, the volume of EDs. To overcome this shortcoming, the model with one warped ED and two branes, known as RS1, was proposed by Randall and Sundrum (RS) [58].

The RS1 model is described by the background warped metric

$$
d s^{2}=e^{-2 \sigma(y)} \eta_{\mu \nu} d x^{\mu} d x^{\nu}-d y^{2},
$$

where $\eta_{\mu \nu}$ is the Minkowski tensor with the signature $(+,-,-,-)$ and $y$ is an extra coordinate. The periodicity condition $y=y+2 \pi r_{c}$ is imposed, and the points $\left(x_{\mu}, y\right)$ and $\left(x_{\mu},-y\right)$ are identified. As a result, we have a model of gravity in a slice of the five-dimensional anti-de Sitter space-time $\mathrm{AdS}_{5}$ compactified to the orbifold $S^{1} / Z_{2}$. The orbifold has two fixed points, $y=0$ and $y=\pi r_{c}$. Two branes are located at these points (called Planck and TeV brane). All the SM fields are assumed to live on the $\mathrm{TeV}$ brane.

The classical action of the RS1 model is [58]

$$
\begin{aligned}
S= & \int d^{4} x \int_{-\pi r_{c}}^{\pi r_{c}} d y \sqrt{G}\left(2 \bar{M}_{5}^{3} \mathcal{R}-\Lambda\right) \\
& +\int d^{4} x \sqrt{\left|g^{(1)}\right|}\left(\mathcal{L}_{1}-\Lambda_{1}\right)+\int d^{4} x \sqrt{\left|g^{(2)}\right|}\left(\mathcal{L}_{2}-\Lambda_{2}\right),
\end{aligned}
$$

where $G_{M N}(x, y)$ is the five-dimensional metric, $M, N=0$, $1,2,3,4$. The quantities $g_{\mu \nu}^{(1)}(x)=G_{\mu \nu}(x, y=0)$, $g_{\mu \nu}^{(2)}(x)=G_{\mu \nu}\left(x, y=\pi r_{c}\right)$, where $\mu=0, \quad 1,2, \quad 3$, are induced metrics on the branes, $\mathcal{L}_{1}$ and $\mathcal{L}_{2}$ are brane Lagrangians, $G=\operatorname{det}\left(G_{M N}\right)$, and $g^{(i)}=\operatorname{det}\left(g_{\mu \nu}^{(i)}\right)$ $(i=1,2) . \bar{M}_{5}$ is the reduced five-dimensional Planck scale, $M_{5} /(2 \pi)^{1 / 3}, M_{5}$ being the fundamental gravity scale in five dimensions. $\Lambda$ is a five-dimensional cosmological constant, while $\Lambda_{1,2}$ are brane tensions.

The warp function $\sigma(y)$ in Eq. (14) obeys EinsteinHilbert's equations. For the first time, it was derived in Ref. [58] to be $\sigma_{\mathrm{RS}}(y)=\kappa|y|$, where $\kappa$ is a parameter with a dimension of mass. It defines the curvature of the fivedimensional space-time, $\mathcal{R}=-20 \kappa^{2}$.

The hierarchy relation in the RS1 model is of the form [58]

$$
\bar{M}_{\mathrm{Pl}}^{2}=\left.\frac{\bar{M}_{5}^{3}}{\kappa}\left[1-e^{-2 \pi \kappa r_{c}}\right]\right|_{\kappa \pi r_{c} \gg 1}=\frac{\bar{M}_{5}^{3}}{\kappa} .
$$

For this relation to be satisfied, one has to put $\bar{M}_{5} \sim \kappa \sim \bar{M}_{\mathrm{Pl}}$. It was shown that $0.01<\kappa / \bar{M}_{5}<0.1$ [59]. As a result, experimental signature of the RS1 model is a series of heavy resonances, with masses defined by the formula

$$
m_{n}=x_{n} \kappa e^{-\pi \kappa r_{c}}, \quad n=1,2, \ldots,
$$

where $x_{n}$ are zeros of the Bessel function $J_{1}(x)$.

In Ref. [60], a general solution for $\sigma(y)$ was derived. It looks like

$$
\begin{aligned}
\sigma(y)= & \frac{\kappa r_{c}}{2}\left[\left|\operatorname{Arccos}\left(\cos \frac{y}{r_{c}}\right)\right|-\left|\pi-\operatorname{Arccos}\left(\cos \frac{y}{r_{c}}\right)\right|\right] \\
& +\frac{\pi|\kappa| r_{c}}{2}-C
\end{aligned}
$$

where $\operatorname{Arccos}(z)$ is a principal value of the multivalued inverse trigonometric function $\arccos (z)$ and $C$ is a 
$y$-independent arbitrary parameter. By taking $C=0$ in (18), we reproduce the RS1 model; while putting $C=\pi \kappa r_{c}$, we come to the Randall-Sundrum-like scenario with a small curvature of the space-time (RSSC) model (for details, see Refs. [61,64]). It was applied for exploring a number of processes at the LHC $[65,66]$.

Let us see what the main features of the RSSC model are in comparison with the features of the RS1 model. The interactions of the KK gravitons $h_{\mu \nu}^{(n)}$ with the SM fields on the $\mathrm{TeV}$ brane are given by the effective Lagrangian density

$$
\begin{aligned}
\mathcal{L}_{\text {int }}= & -\frac{1}{\bar{M}_{\mathrm{Pl}}} h_{\mu \nu}^{(0)}(x) T_{\alpha \beta}(x) \eta^{\mu \alpha} \eta^{\nu \beta} \\
& -\frac{1}{\Lambda_{\pi}} \sum_{n=1}^{\infty} h_{\mu \nu}^{(n)}(x) T_{\alpha \beta}(x) \eta^{\mu \alpha} \eta^{\nu \beta},
\end{aligned}
$$

where $T^{\mu \nu}(x)$ is the energy-momentum tensor of the SM fields (recall that all SM fields are confined on the TeV brane). The coupling constant is given as

$$
\Lambda_{\pi}=\left(\frac{\bar{M}_{5}^{3}}{\kappa}\right)^{1 / 2}
$$

In the RSSC model, the hierarchy relation takes the form

$$
\bar{M}_{\mathrm{Pl}}^{2}=\left.\frac{\bar{M}_{5}^{3}}{\kappa}\left[e^{2 \pi \kappa r_{c}}-1\right]\right|_{\kappa \pi r_{c} \gg 1}=\frac{\bar{M}_{5}^{3}}{\kappa} e^{2 \pi \kappa r_{c}} .
$$

This relation should be compared with Eq. (16). The masses of the KK gravitons are equal to [61,62]

$$
m_{n}=x_{n} \kappa, \quad n=1,2, \ldots
$$

If we take $\kappa \ll \bar{M}_{5} \sim 1 \mathrm{TeV}$, we obtain an almost continuous graviton mass spectrum, which is similar to the spectrum of the ADD model (13), since $\Delta m_{K K} \simeq \pi \kappa$. Let us recall that in the RS1 model the KK gravitons are heavy resonances with masses above few $\mathrm{TeV}$.

Since in the RSSC scenario the warp factor $e^{-2 \sigma(y)}$ is equal to unity on the $\mathrm{TeV}$ brane $\left(y=\pi r_{c}\right)$, the coordinates on this brane are Galilean, and the four-dimensional graviton field $h_{\mu \nu}^{(n)}(x)$ couples to energy-momentum of the ordinary matter $T_{\mu \nu}(x)$ in the usual way [67]. The Einstein tensor $R_{\mu \nu}-(1 / 2) R g_{\mu \nu}$ is invariant under transformation $\sigma(y) \rightarrow \sigma(y)-C$. As for the energy-momentum tensor, it is invariant only for massless fields. The invariance of the gravity action under such transformation needs rescaling of the graviton fields and their masses: $h_{\mu \nu}^{(n)}=e^{-C} h_{\mu \nu}^{\prime(n)}, m_{n}=e^{-C} m_{n}^{\prime}$. We see that the theory of massive KK gravitons is not scale invariant. Only its zero mass sector (standard gravity) remains unchanged. More details can be found in Ref. [60].
Sometimes, it is convenient to work with a conformally flat metric by introducing the coordinate $z=\kappa^{-1} e^{\sigma(y)}$ [68]. Then, the reduced Planck scale reads $\bar{M}_{\mathrm{Pl}}^{2}=$ $\left(\bar{M}_{5} / \kappa\right)^{3}\left(z_{1}^{-2}-z_{2}^{-2}\right)$, and the $\mathrm{KK}$ graviton mass is given as $m_{m}=x_{n} z_{2}^{-1}$, where $z_{1}\left(z_{2}\right)$ is the conformal coordinate of the Planck (TeV) brane. In the RS1 model, $z_{1} \kappa=1$, and $z_{2} \kappa=e^{\pi \kappa r_{c}}$ [see, correspondingly, Eqs. (16) and (17)]. On the contrary, in the RSSC model, $z_{1} \kappa=e^{-\pi \kappa r_{c}}$, and $z_{2} \kappa=1$ [see Eqs. (21) and (22)]. Note that the exponential hierarchy between the branes is the same in both models, $z_{2} / z_{1}=e^{\pi \kappa r_{c}}$.

Now, let us consider the $s$-channel KK graviton exchange contribution to the matrix element of the subprocess $\gamma \gamma \rightarrow \gamma \gamma$ with the invariant energy $\sqrt{\hat{s}}$. It is defined by the formula

$$
\begin{aligned}
M_{K K}= & \frac{1}{2 \Lambda_{\pi}^{2}} \sum_{n=1}^{\infty} e_{\gamma}\left(p_{1}\right) e_{\delta}\left(p_{2}\right) \Gamma^{\mu \nu \gamma \delta}\left(p_{1}, p_{2}\right) \frac{B_{\mu \nu \alpha \beta}}{\hat{s}-m_{n}^{2}+i \Gamma_{n}} \\
& \left.\times \Gamma^{\alpha \beta \rho \sigma}\left(k_{1}, k_{2}\right) e_{\rho}\left(k_{1}\right) e_{\sigma}\left(k_{2}\right)\right],
\end{aligned}
$$

where $k_{i}, p_{i}(i=1,2)$ are momenta of incoming and outgoing photons, while $e_{\mu}\left(k_{i}\right), e_{\mu}\left(p_{i}\right)$ are their polarization vectors. $\Gamma^{\alpha \beta \rho \sigma}$ is a $h^{(n)} \gamma \gamma$ vertex function, and $B_{\mu \nu \alpha \beta}$ is a tensor part of the graviton propagator. Explicit forms of the tensors $\Gamma^{\alpha \beta \rho \sigma}$ and $B_{\mu \nu \alpha \beta}$ can be found in Ref. [44]. The coherent sum in (23) is over KK modes. The total width of the graviton with the KK number $n$ and mass $m_{n}$ is given by $\Gamma_{n}=0.09 m_{n}^{3} / \Lambda_{\pi}^{2}$ [63].

Let us concentrate on the scalar part of the sum (23), which is universal for all types of processes mediated by the s-channel exchanges of the KK gravitons. It is of the form

$$
\mathcal{S}(s)=\frac{1}{\Lambda_{\pi}^{2}} \sum_{n=1}^{\infty} \frac{1}{s-m_{n}^{2}+i \Gamma_{n}} .
$$

This sum has been calculated in Ref. [64],

$$
\mathcal{S}(s)=-\frac{1}{4 \bar{M}_{5}^{3} \sqrt{s}} \frac{\sin (2 A)+i \sinh (2 \varepsilon)}{\cos ^{2} A+\sinh ^{2} \varepsilon},
$$

where

$$
A=\frac{\sqrt{s}}{\kappa}, \quad \varepsilon=0.045\left(\frac{\sqrt{s}}{\bar{M}_{5}}\right)^{3} .
$$

As for the contribution from the $t$-channel graviton exchanges, $\mathcal{S}(\hat{t})$, is was shown in Ref. [64] that the function $\mathcal{S}(t)$ is pure real for $t<0, \bar{M}_{5} \gg \kappa$,

$$
\mathcal{S}(t)=-\frac{1}{2 \bar{M}_{5}^{3} \sqrt{-t}} .
$$

Analogously, we have for the $u$-channel graviton exchanges 


$$
\mathcal{S}(u)=-\frac{1}{2 \bar{M}_{5}^{3} \sqrt{-u}} .
$$

Let us underline that a magnitude of the matrix element is defined by the fundamental gravity scale $\bar{M}_{5}$ and not by the coupling constant $\Lambda_{\pi}$ (20).

The virtual KK graviton exchanges should lead to deviations from the SM predictions both in a magnitude of the cross sections and angular distribution of the final photons because of the spin-2 nature of the gravitons. For the ADD model, the pure KK graviton contribution to the matrix element of the subprocess $\gamma \gamma \rightarrow \gamma \gamma$ was calculated in Ref. [51]. Its generalization for the RSSC model looks like

$$
\begin{aligned}
\left|M_{K K}\right|^{2}= & \frac{1}{8}\left\{|\mathcal{S}(\hat{s})|^{2}\left(\hat{t}^{4}+\hat{u}^{4}\right)+|\mathcal{S}(\hat{t})|^{2}\left(\hat{s}^{4}+\hat{u}^{4}\right)\right. \\
& +|\mathcal{S}(\hat{u})|^{2}\left(\hat{s}^{4}+\hat{t}^{4}\right)+\left[\mathcal{S}(\hat{s})^{\star} \mathcal{S}(\hat{t})\right. \\
& \left.+\mathcal{S}(\hat{s}) \mathcal{S}^{\star}(\hat{t})\right] \hat{u}^{4}+\left[\mathcal{S}(\hat{s})^{\star} \mathcal{S}(\hat{u})+\mathcal{S}(\hat{s}) \mathcal{S}^{\star}(\hat{u})\right] \hat{t}^{4} \\
& \left.+\left[\mathcal{S}^{\star}(\hat{t}) \mathcal{S}(\hat{u})+\mathcal{S}(\hat{t}) \mathcal{S}^{\star}(\hat{u})\right] \hat{s}^{4}\right\},
\end{aligned}
$$

where $\hat{s}, \hat{t}$, and $\hat{u}$ are Mandelstam variables of the subprocess $\gamma \gamma \rightarrow \gamma \gamma$ and the functions $\mathcal{S}(s), \mathcal{S}(t)$, and $\mathcal{S}(u)$ are defined above.

\section{NUMERICAL ANALYSIS}

As was mentioned above, in the RSSC model, the KK graviton spectrum is similar to that in the ADD model. That is why, in contrast to the original RS1 model, an account of effects from EDs in the RSSC model leads to deviations from the SM in magnitudes both of differential cross sections and total cross sections for the photon-induced process $p p \rightarrow p \gamma \gamma p \rightarrow p^{\prime} \gamma \gamma p^{\prime}$ at the LHC. This process goes via electroweak subprocess $\gamma \gamma \rightarrow \gamma \gamma$.

Our main goal is to calculate these deviations as a function of the parameters of the RSSC model. It will enable us to set the $95 \%$ C.L. search limits for the reduced five-dimensional Planck scale $\bar{M}_{5}$. Let us underline that these limits do not depend (up to small power corrections proportional to $\kappa / \bar{M}_{5}$ ) on a value of the second parameter of the model $\kappa$. It is an interesting feature of the RS-like scenario with the small curvature.

Since we impose the cut $W>200 \mathrm{GeV}$ on the diphoton invariant mass, we can neglect the QCD loop contributions (see the Introduction). In fact, $200 \mathrm{GeV}$ is the minimal accessible mass for the present acceptance $\xi=0.015$ of the forward detectors in ATLAS (AFP) or CMS-TOTEM (CT-PPS). However, going to diproton masses (or diphoton masses, since this is the same for exclusive events) below $350-400 \mathrm{GeV}$ would not be easy and would possibly require the installation of new detectors. Below, to estimate the LHC search limit, we will take the cut $p_{t}>$ $300(500) \mathrm{GeV}$, where $p_{t}$ is the final photon transverse momentum. Note that $W \geq 2 p_{t}$ due to energy conservation.
Thus, the condition $W>200 \mathrm{GeV}$ will be automatically satisfied.

We also impose the cut $\left|\eta_{p p}^{i}\right|<2.5$ on the rapidities of the final photons $\eta_{p p}^{i}(i=1,2)$ in the center-of-mass system (c.m.s) of the colliding protons. It is equivalent to the inequality

$$
\eta_{\gamma \gamma}+\left|\eta_{X}\right|<2.5
$$

where

$$
\eta_{\gamma \gamma}=\ln \frac{W+\sqrt{W^{2}-4 p_{t}^{2}}}{2 p_{t}}
$$

is the rapidity of the final photons in the c.m.s of two photons and

$$
\eta_{X}=\frac{1}{2} \ln \frac{\xi_{1}}{\xi_{2}}
$$

is the rapidity of the diphoton system in the in the c.m.s of the incoming protons.

We will also consider the acceptance $0.015<\xi<0$. 5 . It is not yet achievable at the LHC because it goes beyond the collimator acceptance and suffers from very large proton dissociation correction, but we present some results for this acceptance for comparison with the acceptance $0.015<\xi<0.15$.

The results of our calculations of the differential cross sections $d \sigma / d p_{t}$ with the cuts mentioned above as a function of the photon transverse momenta are presented in Figs. 3 and 4 for three values of $\bar{M}_{5}$. Our calculations have shown that the differential cross section does not

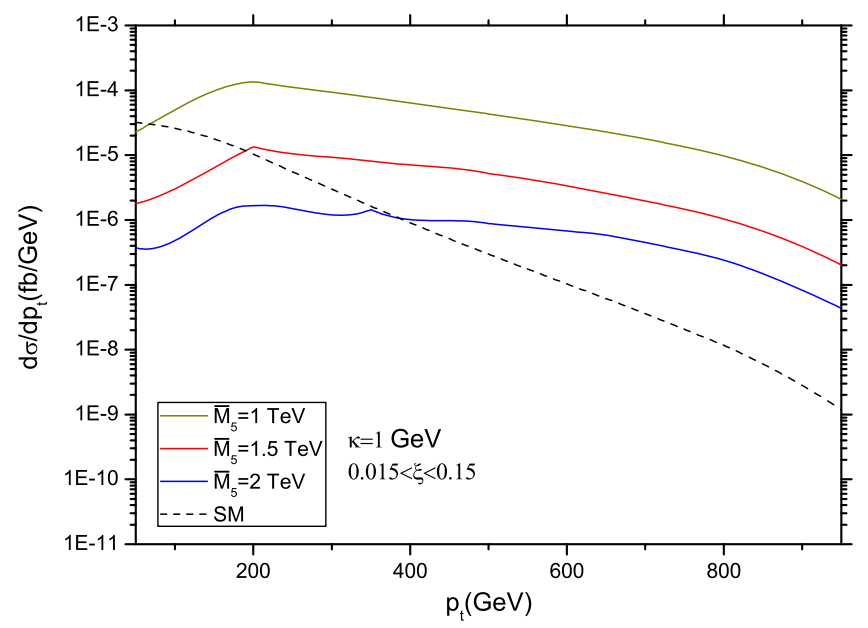

FIG. 3. The differential cross section for the process $p p \rightarrow$ p $\gamma p$ as a function of the transverse momenta of the final photons for $\kappa=1 \mathrm{GeV}$ and for the acceptance region $0.015<\xi<0.15$. The cut on the photon rapidities, $|\eta|<2.5$, is imposed. Here and below, the dotted line denotes the SM contribution. 


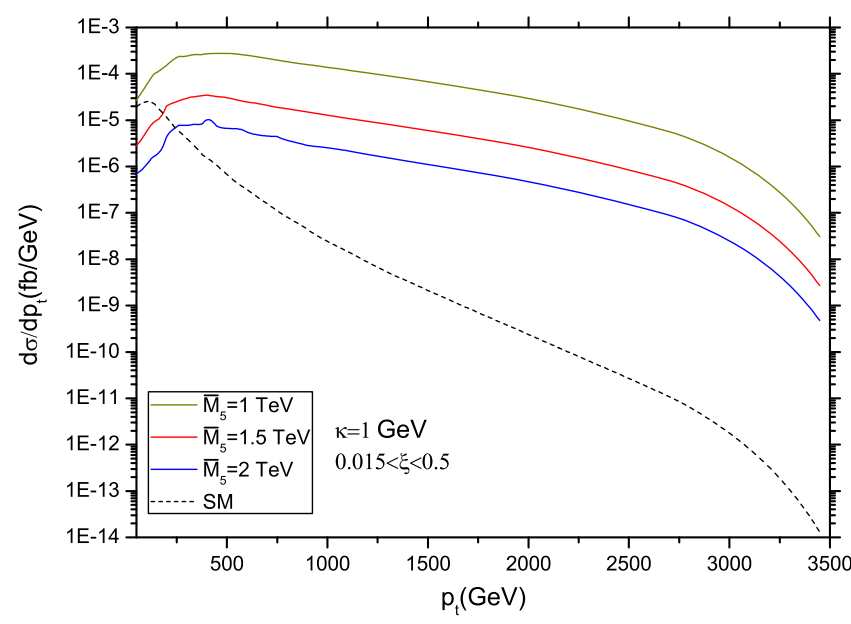

FIG. 4. The same as in Fig. 3, but for the acceptance region $0.015<\xi<0.5$.

practically depend on the curvature parameter $\kappa$. The same is true for the dimuon production in photon-induced events at the LHC [44]. One can see that $d \sigma / d p_{t}$ exceeds the SM cross section $d \sigma_{\mathrm{SM}} / d p_{t}$ for $p_{t}>300 \mathrm{GeV}$, if $0.015<$ $\xi<0.15$, and for $p_{t}>500 \mathrm{GeV}$, if $0.015<\xi<0.5$. Moreover, the difference between $d \sigma / d p_{t}$ and $d \sigma_{\mathrm{SM}} / d p_{t}$ increases as $p_{t}$ grows. The effect is more pronounced for smaller values of $\bar{M}_{5}$. The maximum of $d \sigma / d p_{t}$ around $p_{t} \simeq 200 \mathrm{GeV}(500 \mathrm{GeV})$ for the acceptance region $0.015<\xi<0.15(0.015<\xi<0.5)$ is a result of the integration in variable $W$, the lower limit of which depends on $p_{t}$, as well as due to the $p_{t}$ dependence of the rapidity cut (30)-(31).

The total cross section $\sigma\left(p_{t}>p_{t, \text { min }}\right)$ for two acceptance regions is shown in Figs. 5 and 6 as a function of the minimal transverse momenta of the final photons $p_{t, \min }$.

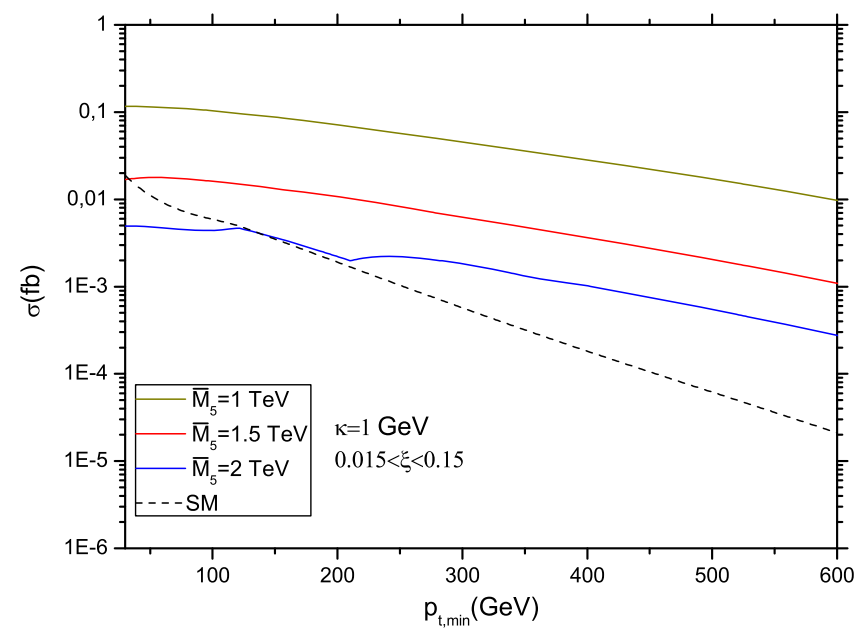

FIG. 5. The total cross section for the process $p p \rightarrow p \gamma \gamma p$ as a function of the minimal transverse momenta of the final photons $p_{t \text {,min }}$ for the acceptance region $0.015<\xi<0.15$ for different values of $\bar{M}_{5}$.

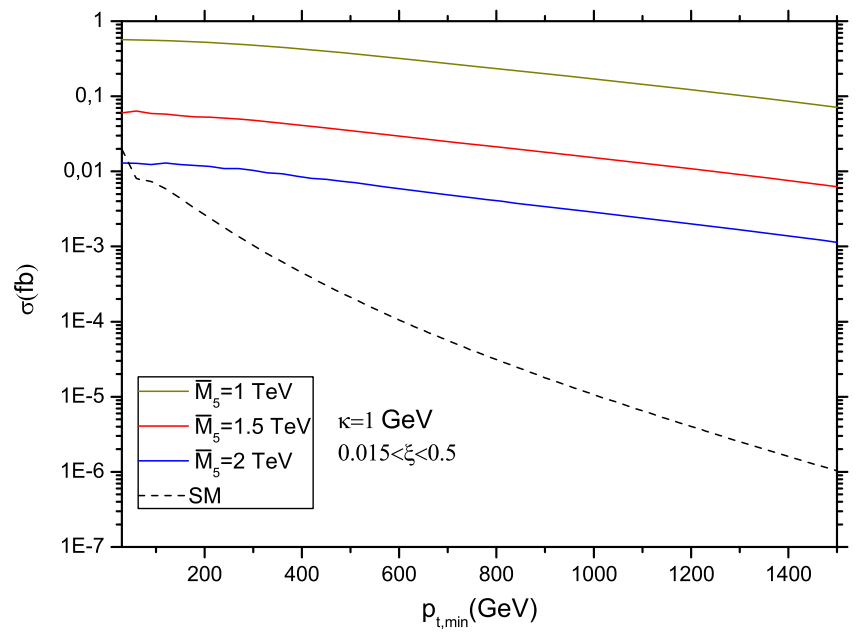

FIG. 6. The same as in Fig. 5, but for the acceptance region $0.015<\xi<0.5$.

In both figures, the comparison with the pure SM predictions is given. For both acceptance regions, a deviation of $\sigma\left(p_{t}>p_{t, \min }\right)$ from the SM cross section $\sigma_{\mathrm{SM}}\left(p_{t}>\right.$ $\left.p_{t, \text { min }}\right)$ gets higher as $p_{t, \text { min }}$ grows. The effect is more significant for $0.015<\xi<0.5$.

Having calculations of the total cross sections in hand, we are able to obtain the limits on $\bar{M}_{5}$ for two acceptance regions, $0.015<\xi<0.15$ and $0.015<\xi<0.5$, for $p_{t}>$ $300 \mathrm{GeV}$ and $p_{t}>500 \mathrm{GeV}$, respectively. In sensitivity analysis, we use the likelihood method from Ref. [40]. We assume that observed events follow a Poisson distribution. Then, the statistics together with the prediction of the event rate leads to the following likelihood function:

$$
\mathfrak{L}(\sigma)=\operatorname{Pr}(\mathrm{n} \mid \mathrm{b}+\sigma \mathrm{L}) .
$$

Here, $n$ is the number of the observed events, $b$ is the expected number of background (SM) events, $\sigma$ is the total cross section, and $L$ is the integrated luminosity. One can estimate from Figs. 5 and 6 that for the maximum luminosity value of $L=300 \mathrm{fb}^{-1}\left(L=3000 \mathrm{fb}^{-1}\right)$ when $p_{t}>300 \mathrm{GeV}\left(p_{t}>500 \mathrm{GeV}\right)$ the expected number of the SM events is less than 0.5 . Thus, we can assume that no events is observed and put $b=0$. Then, the LHC exclusion region for the credibility $1-\alpha$ is given by the formula [40]

$$
\sigma_{\alpha}=-\frac{1}{L} \ln (\sigma)
$$

For the $95 \%$ C.L., which corresponds to $\alpha=0.05$, we get from Eq. (34)

$$
\sigma_{0.05} \simeq \frac{3}{L}
$$

First, let us consider the acceptance region $0.015<\xi<$ 0.15 and impose the cut $p_{t}>300 \mathrm{GeV}$. Using Eq. (34), we 


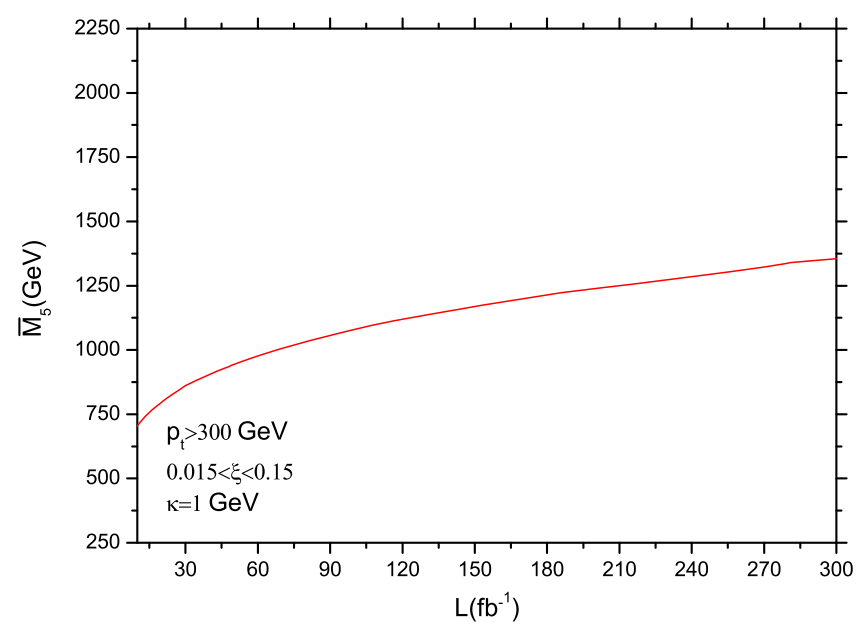

FIG. 7. The 95\% C.L. search limits for the reduced fivedimensional gravity scale $\bar{M}_{5}$ as a function of the integrated LHC luminosity with $p_{t}>300 \mathrm{GeV}$ for the acceptance region $0.015<\xi<0.15$. The rapidity cut of 2.5 on the photon rapidities is imposed.

have found the $95 \%$ C.L. search limits for the reduced fivedimensional gravity scale $\bar{M}_{5}$ as a function of the integrated LHC luminosity; see Fig. 7. The analogous results for the cut $p_{t}>500 \mathrm{GeV}$ are presented in Fig. 8. As one can see, for the integrated luminosity $L=300 \mathrm{fb}^{-1}$, the sensitivity bound on $\bar{M}_{5}$ is $1.37 \mathrm{TeV}$ for the $0.015<\xi<0.15$. For $L=3000 \mathrm{fb}^{-1}$, the sensitivity bound on $\bar{M}_{5}$ is equal to $1.74 \mathrm{TeV}$ for this acceptance region. Let us underline that these bound do not depend on the parameter $\kappa$, provided $\kappa \ll \bar{M}_{5}$, which is satisfied in our analysis.

Our bounds on the five-dimensional gravity scale $\bar{M}_{5}$ are rather low in comparison with the experimental bounds on $D$-dimensional scale $M_{D}$ in the ADD model (see, for instance, Ref. [69]). In this regard, we must emphasize that the LHC bounds on $M_{D}$ cannot be directly applied to the gravity scale $\bar{M}_{5}$ in the RSSC model. As was mentioned

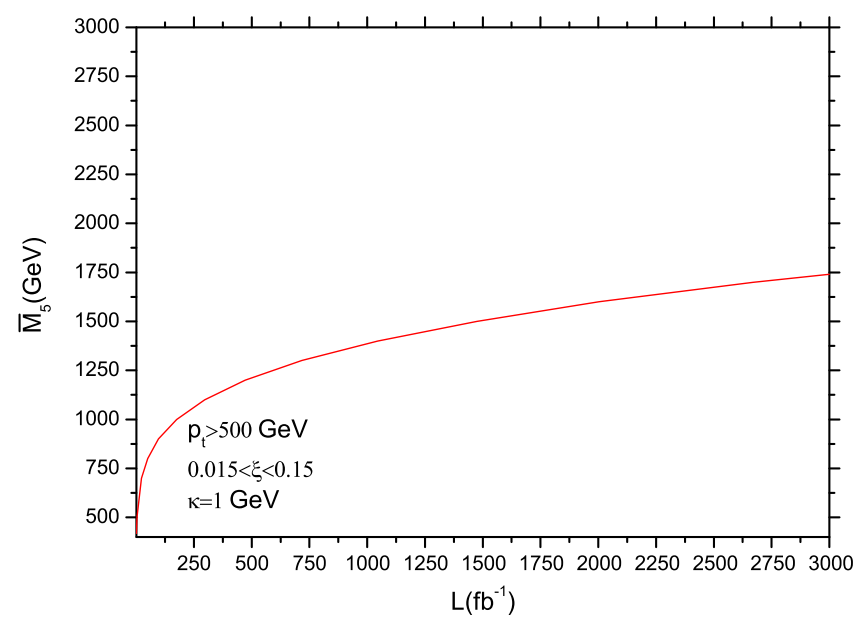

FIG. 8. The same as in Fig. 7, but for $p_{t}>500 \mathrm{GeV}$. above (for details, see Ref. [64]), this model cannot be regarded as a small distortion of the ADD model even for very small values of the curvature $\kappa$. Moreover, in the ADD model, the number of EDs should be $d \geq 2$, while in the RSSC model, we deal with one ED. As for the original RS1 model, the bounds in it are put on the set of two parameters: the ratio $\kappa / \bar{M}_{5}$ and $m_{1}$, which is the mass of the lightest KK graviton.

We consider the diphoton production in the photoninduced process at the LHC as a mean of looking for effects of low gravity scale $\bar{M}_{5}$ in the Randall-Sundrum-like scenario with the small curvature.

\section{CONCLUSIONS}

With the forward detectors prepared by the ATLAS Forward Physics Collaboration and CMS-TOTEM Precision Proton Spectrometer Collaboration [9-12], it becomes possible to investigate the exclusive photoninduced process $p p \rightarrow p \gamma \gamma p \rightarrow p^{\prime} X p^{\prime}$ (see Fig. 1). In the present paper, we have studied the diphoton production $p p \rightarrow p \gamma \gamma p \rightarrow p^{\prime} \gamma \gamma p^{\prime}$ at the LHC energy $14 \mathrm{TeV}$ in the framework of the Randall-Sundrum-like model with one warped ED and small curvature of the five-dimensional space-time. The consideration was performed basically for an acceptance region of the forward detector, $0.015<$ $\xi<0.15$. Extra dimension contributions are greater at higher energies. Therefore, we also examine the acceptance $0.015<\xi<0.5$ (it is not yet possible at the LHC as described above) to make a comparison with the acceptance range $0.015<\xi<0.15$. Here, $\xi$ is the fractional proton momentum loss of the incident protons.

For a background, we have considered the SM diphoton contribution, which is negligible. The highest background is two photon plus protons originating from pile-up [70], but with the luminosity $3000 \mathrm{fb}^{-1}$, it is no longer zero, but still very small.

The distributions in the photon transverse momenta $p_{t}$ with the cut $|\eta|<2.5$ imposed on the photon rapidity $\eta$ have been calculated as a function of the reduced fivedimensional Planck scale $\bar{M}_{5}$ (see Figs. 3 and 4). It was shown that the deviation from the SM predictions gets higher as $p_{t}$ grows. The total cross sections have been calculated for two acceptance regions depending on the cut imposed on the transverse momenta of the final photon, $p_{t}>p_{t, \text { min }}$ (see Figs. 5 and 6). Let us underline that in the RSSC model the values of the cross sections do not depend on the curvature parameter $\kappa$, provided $\kappa \ll \bar{M}_{5}$, which was satisfied in our analysis. This allowed us to put the 95\% C.L. search limits for $\bar{M}_{5}$ as a function of the integrated LHC luminosity (see Figs. 7 and 8). For instance, for $0.015<$ $\xi<0.15$ and $p_{t}>300 \mathrm{GeV}$, this limit for $\bar{M}_{5}$ is equal to $1.37 \mathrm{TeV}$, for the integrated luminosity $L=300 \mathrm{fb}^{-1}$. For the HL-LHC integrated luminosity $L=3000 \mathrm{fb}^{-1}$ and $p_{t}>500 \mathrm{GeV}$, we have found that the $95 \%$ C.L. 
search limit is equal to $1.74 \mathrm{TeV}$, for the same acceptance region. Any BSM scenario must be investigated in a variety of processes in order to find the most appropriate one. Recently, the dimuon production in the photon-induced process at the LHC was studied in Ref. [44], in which search limits for $\bar{M}_{5}$ have been also obtained. The bounds on $\bar{M}_{5}$ in the present article are better than the bounds obtained in Ref. [44].
[1] T. Aaltonen et al. (CDF Collaboration), Phys. Rev. Lett. 102, 242001 (2009).

[2] T. Aaltonen et al. (CDF Collaboration), Phys. Rev. Lett. 102, 222002 (2009).

[3] S. Chatrchyan et al. (CMS Collaboration), J. High Energy Phys. 01 (2012) 052.

[4] S. Chatrchyan et al. (CMS Collaboration), J. High Energy Phys. 11 (2012) 080.

[5] G. Aad et al. (ATLAS Collaboration), Phys. Lett. B 749, 242 (2015).

[6] G. Aad et al. (ATLAS Collaboration), J. High Energy Phys. 08 (2016) 009.

[7] M. Aaboud et al. (ATLAS Collaboration), Phys. Rev. D 94, 032011 (2016).

[8] A. M. Sirunyan et al. (CMS and TOTEM Collaborations), J. High Energy Phys. 07 (2018) 153.

[9] ATLAS Collaboration, CERN Technical Report No. CERNLHCC-2011-012; CERN Technical Report No. LHCC-I020, 2011.

[10] L. Adamczyk et al., CERN Technical Report No. ATLCOMLUM-2011-006, 2011.

[11] L. Adamczyk et al., CERN Technical Report No. CERNLHCC-2015-009; CERN Tehcnical Report No. ATLASTDR-024, 2015.

[12] M. Albrow et al., CERN Technical Report No. CERNLHCC-2014-021; CERN Technical Report No. TOTEMTDR-003; CERN Technical Report No. CMS-TDR-13, 2014.

[13] G. Antchev et al. (TOTEM Collaboration), Europhys. Lett. 96, 21002 (2011).

[14] G. Antchev et al. (TOTEM Collaboration), Europhys. Lett. 98, 31002 (2012).

[15] G. Antchev et al. (TOTEM Collaboration), Europhys. Lett. 101, 21002 (2013).

[16] M. Albrow et al. (FP420 R and D Collaboration), J. Instrum. 4, T10001 (2009).

[17] M. G. Albrowa, T. D. Coughlin, and J. R. Forshaw, Prog. Part. Nucl. Phys. 65, 149 (2010).

[18] K. Piotrzkowski, Phys. Rev. D 63, 071502(R) (2001).

[19] V. Goncalves and M. Machado, Phys. Rev. D 75, 031502(R) (2007).

[20] İ. Şahin and S. C. İnan, J. High Energy Phys. 09 (2009) 069.

[21] S. C. İnan, Phys. Rev. D 81, 115002 (2010).

[22] S. Atağ and A. Billur, J. High Energy Phys. 11 (2010) 060.

[23] İ. Şahin and A. A. Billur, Phys. Rev. D 83, 035011 (2011).

[24] İ. Şahin and M. Köksal, J. High Energy Phys. 11 (2011) 100.

[25] S. C. İnan and A. A. Billur, Phys. Rev. D 84, 095002 (2011).
[26] R. S. Gupta, Phys. Rev. D 85, 014006 (2012).

[27] İ. Şahin, Phys. Rev. D 85, 033002 (2012).

[28] L. N. Epele, H. Fanchiotti, C. A. G. Canal, V. A. Mitsou, and V. Vento, Eur. Phys. J. Plus 127, 60 (2012).

[29] İ. Şahin and B. Şahin, Phys. Rev. D 86, 115001 (2012).

[30] A. A. Billur, Europhys. Lett. 101, 21001 (2013).

[31] İ. Şahin, A. A. Billur, S. C. İnan, B. Şahin, M. Köksal, P. Tektaş, E. Alıcı, and R. Yıldırım, Phys. Rev. D 88, 095016 (2013).

[32] H. Sun and C. X. Yue, Eur. Phys. J. C 74, 2823 (2014).

[33] H. Sun, Nucl. Phys. B886, 691 (2014).

[34] İ. Şahin, M. Köksal, S. C. İnan, A. A. Billur, B. Şahin, P. Tektaş, E. Alıcı, and R. Yıldırım, Phys. Rev. D 91, 035017 (2015).

[35] M. Köksal and S. C. İnan, Adv. High Energy Phys. 2014, 315826 (2014).

[36] S. Fichet, G. von Gersdorff, and C. Royon, Phys. Rev. Lett. 116, 231801 (2016).

[37] S. Fichet, G. von Gersdorff, and C. Royon, Phys. Rev. D 93, 075031 (2016).

[38] S. Fichet, J. High Energy Phys. 04 (2017) 088.

[39] C. Baldenegro, S. Fichet, G. von Gersdorff, and C. Royon, J. High Energy Phys. 06 (2017) 142.

[40] C. Baldenegro, S. Fichet, G. von Gersdorff, and C. Royon, J. High Energy Phys. 06 (2018) 131.

[41] M. Köksal, S. C. İnan, A. A. Billur, Y. Özgüven, and M. K. Bahar, Phys. Lett. B 783, 375 (2018).

[42] M. Aaboud et al. (ATLAS Collaboration), Nat. Phys. 13, 852 (2017).

[43] D. d'Enterria et al. (CMS Collaboration), Nucl. Phys. A982, 791 (2019).

[44] S. C. İnan and A. V. Kisselev, Eur. Phys. J. C 78, 729 (2018).

[45] R. Karplus and M. Neuman, Phys. Rev. 83, 776 (1951).

[46] V. Costantini, B. De Tollis, and G. Pistoni, Nuovo Cimento A 2, 733 (1971).

[47] G. Jikia and A. Tkabladze, Phys. Lett. B 323, 453 (1994).

[48] G. J. Gounaris, P. I. Porfyriadis, and F. M. Renard, Phys. Lett. B 452, 76 (1999); 513, 431(E) (2001).

[49] G. J. Gounaris, P. I. Porfyriadis, and F. M. Renard, Eur. Phys. J. C 9, 673 (1999).

[50] S. Fichet, G. von Gersdorff, B. Lenzi, C. Royon, and M. Saimpert, J. High Energy Phys. 02 (2015) 165.

[51] S. Atağ, S. C. İnan, and İ. Şahin, J. High Energy Phys. 09 (2010) 042.

[52] V. Budnev, I. Ginzburg, G. Meledin, and V. Serbo, Phys. Rep. 15, 181 (1975).

[53] G. Baur, K. Hencken, D. Trautmann et al., Phys. Rep. 364, 359 (2002). 
[54] J. Polchinski, String Theory (Cambridge University Press, Cambridge, England, 1998), Vols. I and II.

[55] N. Arkani-Hamed, S. Dimopoulos, and G. Dvali, Phys. Lett. B 429, 263 (1998).

[56] N. Arkani-Hamed, S. Dimopoulos, and G. Dvali, Phys. Rev. D 59, 086004 (1999).

[57] I. Antoniadis, N. Arkani-Hamed, S. Dimopoulos, and G. Dvali, Phys. Lett. B 436, 257 (1998).

[58] L. Randall and R. Sundrum, Phys. Rev. Lett. 83, 3370 (1999).

[59] H. Davoudiasl, J. L. Hewett, and T. G. Rizzo, Phys. Rev. D 63, 075004 (2001).

[60] A. V. Kisselev, Nucl. Phys. B909, 218 (2016).

[61] G. F. Giudice, T. Plehn, and A. Strumia, Nucl. Phys. B706, 455 (2005).
[62] A. V. Kisselev and V. A. Petrov, Phys. Rev. D 71, 124032 (2005).

[63] A. V. Kisselev, Eur. Phys. J. C 42, 217 (2005).

[64] A. V. Kisselev, Phys. Rev. D 73, 024007 (2006).

[65] A. V. Kisselev, J. High Energy Phys. 09 (2008) 039.

[66] A. V. Kisselev, J. High Energy Phys. 04 (2013) 025.

[67] V. A. Rubakov, Phys. Usp. 44, 871 (2001).

[68] A. Chamblin, S. W. Hawking, and H. S. Reall, Phys. Rev. D 61, 065007 (2000).

[69] A. M. Sirunyan et al. (CMS Collaboration), Eur. Phys. J. C 78, 789 (2018); Phys. Rev. D 98, 092001 (2018).

[70] S. Fichet, G. von Gersdorff, O. Kepka, B. Lenzi, C. Royon, and M. Saimpert, Phys. Rev. D 89, 114004 (2014); S. Fichet, G. von Gersdorff, B. Lenzi, C. Royon, and M. Saimpert, J. High Energy Phys. 02 (2015) 165. 